\title{
EFFECT OF NPK RATES AND HUMIC ACID APPLICATIONS ON GROWTH OF EGYPTIAN COTTON \\ Seadh, S. E. ${ }^{1}$; M. H. El-Hendi ${ }^{1}$; H. A. Abd El-Aal ${ }^{2}$ and Shaimaa O. S. El-Sayed ${ }^{2}$ \\ 1. Agronomy Department, Faculty of Agriculture, Mansoura University. \\ 2. Cotton Research Institute, Agriculture Research Center, Giza, Egypt.
}

\begin{abstract}
Two field experiments were carried out at Gemmeiza Agric. Res. Station, El - Gharbia Governorate during 2010 and 2011 seasons, to study the effect of four NPK fertilizers rates (100\%, $75 \%, 50 \%$ and $25 \%$ of recommended rate) and application methods of humic acid on growth of the Egyptian cotton cultivar "Giza 86". The recommended rate of NPK was $45 \mathrm{~kg} \mathrm{~N}+22.50 \mathrm{~kg} \mathrm{P} \mathrm{O}_{5}+24.0 \mathrm{~kg} \mathrm{~K} 2 \mathrm{O} / \mathrm{fed}$.. The solutions of humic acid were applied with the concentration of $5 \mathrm{~cm}^{3} /$ litre as a soaking seed for $18 \mathrm{~h}, 50 \mathrm{~cm}^{3} /$ litre as soil application twice, $5 \mathrm{~cm}^{3} / \mathrm{L}$ as a foliar application at 40,55 and 70 days from sowing and without humic acid as a control treatment.

Leaf area index (LAI), total dry weight/plant, crop growth rate (CGR), plant height and number of fruiting branches/plant were significantly increased by increasing NPK rate from 25 up to $100 \%$ of the recommended rate at all sampling dates in both seasons. Also, application of $100 \%$ NPK recorded the highest net assimilation rate (NAR) at the first period (79-100 days) in the two seasons.

Application of humic acid solution resulted in a significant increase in all mention growth measurements (except NAR) compared with control treatment in both seasons. Foliar application of humic acid resulted in a significant increase in leaf area index, total dry weight/plant, crop growth rate, plant height and number of fruiting branches/plant compared with other application methods in both seasons.

The interaction between NPK rate and humic acid application had a significant effect on LAI, total dry weight/plant, CGR and NAR at the most sampling dates in the two seasons. Plants received 100 or $75 \%$ NPK along with foliar application of humic acid produced higher LAI, total dry weight/plant and CGR than those received $25 \%$ NPK and without humic acid at the most sampling dates in both seasons. The relative ranking of the interaction between NPK rate and humic acid application for NAR was inconsistent at the two period in both seasons. None of the interaction had a significant effect on plant height and number of fruiting branches / plant in the two seasons.

It can be concluded that $75 \%$ NPK and foliar application with humic acid could be recommended for optimum dry matter production of the Egyptian cotton cultivar "Giza 86". This result indicated that foliar application of humic acid can be saved $25 \%$ NPK fertilizer without reduce growth of cotton plant.
\end{abstract}

\section{INTRODUCTION}

In Egypt, cotton (Gossypium barbadense L.) is one of the most important crops for both local industry and export. Therefore, increasing cotton yield and reducing cost of production are essential aims for growers, but it usually depends on many factors especially that influence the plant growth. Nutrition manner is considered as one of the most important factors that affecting plant growth. Furthermore, NPK forms are the most important 
plant nutrients limiting plant growth and consequently yield. But, these chemical fertilizers are becoming expensive coupled with their adverse effect on environment (air, water and soil). This has necessitated reorienting the research with reduced rates of inorganic fertilizers. Many investigators studied the important of NPK fertilizer rates. Srinivasan and Venkatesan (2003) found that application of NPK at the recommended dose (60:30:30 kg NPK ha -1 ) by applying $50 \% \mathrm{~N}$ and full $\mathrm{P}$ and $\mathrm{K}$ as basal and remaining at 45 DAS registered the tallest plants and was comparable with application of $75 \%$ $\mathrm{RD}$ by applying full $\mathrm{P}$ as basal and $\mathrm{K}$ and $\mathrm{N}$ in two splits on 45 and $60 \mathrm{DAS}$. Dobermann et al . (2005) found that application of 120:60:60 kg NPK ha-1 resulted in the tallest plants. Reddy et al. (2007) found that cotton hybrid Mallika did not responded to the increase in fertilizer levels (recommended rate of NPK at $120: 60: 60 \mathrm{~kg}$ ha- 1 and at 25 and $50 \%$ of the recommended rate) with regard to number of sympodia/plant. Reddy and Gopinath (2008) found that number of sympodia/plant was not significantly influenced due to application of three nutrient levels (recommended dose of NPK fertilizers (RDF; 120:60:60 kg ha-1), 25\% more than RDF and 50\% more than RDF).

Great attention has been paid to use the organic substances for minimizing the use of chemical fertilizers and, in turn, improving soil texture and fertility and reducing environmental pollution. Humic acid is a constituent of organic matter. It is the most active fraction of humus coupled with fulvic acid. The use of humic acid might permit satisfactory or improved growth under substantially neutral or alkaline conditions. In this concern, El-Gala (1978) found that the addition of the purified humic acid resulted in an increase in the amount of $\mathrm{Fe}, \mathrm{Mn}, \mathrm{Zn}$ and $\mathrm{Cu}$ in soil solution and this increase was related to ability of humic substances to react and form chelating compound with these elements. Chellaiah et al. (2001) found that spraying cotton plants with $1 \%$ humic acid significantly affected plant height and sympodia/plant. Kalaichelvi and Chinnusamy (2005) found that among potassium humate treatments $(20,30$ and $40 \mathrm{~kg}$ ha-1 soil application, $1.0 \%$ seed soaking; three $0.1 \%$ foliar sprayings ; $20 \mathrm{~kg}$ ha- 1 soil application + three $0.1 \%$ foliar sprayings, and $20 \mathrm{~kg}$ ha- 1 soil application $+1.0 \%$ seed soaking + three $0.1 \%$ foliar sprayings), soil application of either 30 or $40 \mathrm{~kg}$ ha-1 recorded the highest number of sympodial branches. Taha et al. (2006) reported that the positive effect of humic acid on the values of available nutrients in the soil related to the ability of humic acid to form chelating compounds with these elements in the soil. Basbag (2008) reported that humic acid are referred to as humic substances and are used as soil conditioners, soil supplements and fertilizer amendments. Also, he found that different humic acid treatments (seed soaking, foliar spray, seed soaking + foliar spray) affected number of sympodial branches and plant height. Emara and Hamoda (2012) found that the tallest cotton plants and the greatest number of sympodia/plant were produced by foliar spraying of humex three times with the rate of $5 \mathrm{~cm} 3 / \mathrm{L}$.

Therefore, this study aimed to study the effect NPK rates and humic acid treatments as well as their interaction on cotton growth attributes under the environmental conditions of El-Gharbia Governorate. 


\section{MATERIALS AND METHODS}

Two field experiments were carried out at El-Gemmeiza Agricultural Research Station, El-Gharbia Governorate during 2010 and 2011 seasons, to study the effect of four NPK rates and application methods of humic acid on growth of the Egyptian cotton cultivar "Giza 86". The preceding crop was the Egyptian clover (Trifolium alexandrinum $\mathrm{L}$ ) in both seasons.

Representative soil samples were taken from the experimental soil sites at the depth of $0-30 \mathrm{~cm}$ from the soil surface and prepared for analysis according to Jackson (1973). Results of chemical analysis are shown in Table 1.

Table 1. Chemical analysis of the experimental soil $(0-30 \mathrm{~cm}$ depth)in 2010 and 2011 seasons

\begin{tabular}{|c|c|c|}
\hline Properties & 2010 & 2011 \\
\hline Texture & Clay loam & Clay loam \\
\hline $\mathrm{pH}$ & 7.8 & 7.8 \\
\hline EC mmhos/cm. & 0.93 & 1.08 \\
\hline \multicolumn{3}{|c|}{ Cations Meq/L } \\
\hline $\mathrm{Ca}$ & 1.65 & 3.60 \\
\hline $\mathrm{Mg}$ & 0.90 & 1.89 \\
\hline $\mathrm{Na}$ & 6.58 & 7.47 \\
\hline $\mathrm{K}$ & 0.24 & 0.35 \\
\hline \multicolumn{3}{|c|}{ Anions Meq/L } \\
\hline $\mathrm{CO}_{3}$ & - & - \\
\hline $\mathrm{HCO}_{3}$ & 2.27 & 2.70 \\
\hline $\mathrm{Cl}$ & 4.32 & 6.61 \\
\hline $\mathrm{SO}_{4}$ & 2.78 & 4.00 \\
\hline Organic matter $\%$ & 1.9 & 1.75 \\
\hline Available N (ppm) & 30.7 & 21.1 \\
\hline Available P (ppm) & 11.8 & 10.7 \\
\hline Exchangeable K (ppm) & 360 & 410 \\
\hline Available Fe (ppm) & 13.3 & 10.4 \\
\hline Available Mn (ppm) & 11.5 & 9.1 \\
\hline Available Zn (ppm) & 2.8 & 2.5 \\
\hline Available Cu (ppm) & 3.5 & 3.1 \\
\hline
\end{tabular}

The experimental design was split plot with four replications. The main plots were assigned to NPK rates, while the sub-plots were assigned to application methods of humic acid. NPK fertilizer was used at rates of 100 , 75,50 and $25 \%$ of the recommended rate. The recommended rate of NPK was $45 \mathrm{~kg} \mathrm{~N}+22.50 \mathrm{~kg} \mathrm{P}{ }_{2} \mathrm{O}_{5}+24.0 \mathrm{~kg} \mathrm{~K} \mathrm{O}_{2} /$ fed.. Inorganic nitrogen fertilizer was applied in the form of ammonium nitrate $(33.5 \% \mathrm{~N})$ with the previous rates in two equal doses after thinning and at the next irrigation. Phosphorus fertilizer was added in the form of calcium superphosphate $\left(15.5 \% \mathrm{P}_{2} \mathrm{O}_{5}\right)$ during land preparation. Potassium fertilizer was added in the form of potassium sulphate $\left(48 \% \mathrm{~K}_{2} \mathrm{O}\right)$ in one dose after thinning.

Humic acid were applied in the form of actosol with the concentration of $5 \mathrm{~cm}^{3} /$ litre as a soaking seed for $18 \mathrm{~h}, 50 \mathrm{~cm}^{3} /$ litre as soil application twice (after sowing and after thinning), $5 \mathrm{~cm}^{3} / \mathrm{L}$ as a foliar application in three times 
at 40,55 and 70 days from sowing and without humic acid as a control treatment.

Humic acid is the active ingredient of actosol product, which contains 1-5-6 NPK and $20 \%$ humic acid, commercially known as potash actosol and manufactured by ARCTICK Inc, Chentilly, VA, USA). The chemical analysis of actosol compound was determined using $\mathrm{BaCl}$ precipitation method as described by Fataftah et al. (2001) and illustrated in Table 2.

Table 2: Chemical analysis of actosol compound.

\begin{tabular}{|c|c|c|}
\hline \multirow{2}{*}{\multicolumn{2}{|c|}{\begin{tabular}{|l|} 
Component \\
$\mathrm{pH}$
\end{tabular}}} & Value \\
\hline & & 7.6 \\
\hline Humic acid & $\%$ & 20 \\
\hline Total nitrogen & $\%$ & 1 \\
\hline Total phosphorus & $\%$ & 5 \\
\hline Total potassium & $\%$ & 6 \\
\hline Total magnesium & $\%$ & 0.07 \\
\hline Total calcium & $\%$ & 0.08 \\
\hline Total chloride & $\%$ & 0.50 \\
\hline Total iron & ppm & 100 \\
\hline Total zinc & ppm & 10 \\
\hline Total manganese & $\mathrm{ppm}$ & 10 \\
\hline Total copper & ppm & 10 \\
\hline Total boron & $\mathrm{ppm}$ & 7 \\
\hline
\end{tabular}

Each sub-plot consisted of 6 ridges in the first season and 7 ridges in the second season each $5 \mathrm{~m}$ long and $0.7 \mathrm{~m}$ in width. Sowing took place on March 30, 2010 and March 31, 2011. Seeds of cotton cultivar "Giza 86" were sown in hills on one side of ridge at the rate of $3-4$ seeds per hill with $25 \mathrm{~cm}$ between hills. Two plants per hill were maintained by thinning at 35 days after sowing. The other cultural practices were carried out as recommended for conventional cotton seeding in the local production district.

Six guarded plants were randomly taken from each plot at 79, 100 and 121 days from sowing (DFS) to determine leaf area and dry weight per plant. Roots of sample plants was removed at the cotyledonary nodes, then the different plant fractions were washed and oven dried to a constant weight at $70 \mathrm{o} \mathrm{C}$. For leaf area measurements, the disk method was used according to Johnson (1967). The growth analysis, viz. leaf area index (LAl), crop growth rate (CGR) in $\mathrm{g} / \mathrm{m} 2 /$ week and nrt assimilation rate (NAR) in $\mathrm{g} / \mathrm{m} 2 /$ week was computed according to the following formulas (Watson 1952):

$\mathrm{LAl}=$ leaf area per plant/unit ground area occupied by one plant.

$\mathrm{CGR}=\mathrm{W}_{2}-\mathrm{W}_{1} / \mathrm{t}_{2}-\mathrm{t}_{1}$

$N A R=\left(W_{2}-W_{1}\right)\left(\log _{e} A_{2}-\log _{e} A_{1}\right) /\left(A_{2}-A_{1}\right)\left(t_{2}-t_{1}\right)$.

Where: $\mathrm{W} 1, \mathrm{~A} 1$ and $\mathrm{W} 2, \mathrm{~A} 2$ refer to dry weight and leaf area at time t1 and t2 in weeks, respectively.

At maturity ten guarded plants were randomly taken from each plot to determine plant height and number of fruiting branches.

The statistical analysis of the obtained data in the two seasons was performed according to Snedecor and Cochran (1980), and the treatments means were compared using LSD at 0.05 level of probability. 


\section{RESULTS AND DISCUSSION}

\section{Effect of NPK rate:}

NPK rates had a substantial effect on LAl (Table 3) and dry weight/plant (Table 4) at 79, 100 and 121 days from sowing (DFS), CGR (Table 5) at the periods of 79-100 and 100-121 DFS, NAR (Table 6) at the first period, only, and plant height and number of fruiting branches/plant at harvest (Table 7) in both seasons.

Table 3: Leaf area index as affected by NPK rates, humic acid treatments and their interaction in 2010 and 2011 seasons.

\begin{tabular}{|c|c|c|c|c|c|c|c|}
\hline \multirow{3}{*}{\multicolumn{2}{|c|}{ Treatments }} & \multirow{2}{*}{\multicolumn{3}{|c|}{$\begin{array}{c}2010 \text { season } \\
\text { Days from sowing }\end{array}$}} & \multirow{2}{*}{\multicolumn{3}{|c|}{$\begin{array}{c}2011 \text { season } \\
\text { Days from sowing }\end{array}$}} \\
\hline & & & & & & & \\
\hline & & 79 & 100 & 121 & 79 & 100 & 121 \\
\hline \multicolumn{8}{|c|}{ A- NPK rates: } \\
\hline 100 & & $2.505 \mathrm{a}$ & $2.791 \mathrm{a}$ & $3.053 \mathrm{a}$ & $1.994 \mathrm{a}$ & $2.429 \mathrm{a}$ & $2.872 \mathrm{a}$ \\
\hline 75 & & $2.412 \mathrm{~b}$ & $2.679 \mathrm{~b}$ & $2.898 \mathrm{~b}$ & $1.809 \mathrm{~b}$ & $2.327 \mathrm{~b}$ & $2.726 \mathrm{~b}$ \\
\hline 50 & & $2.371 \mathrm{~b}$ & $2.567 \mathrm{c}$ & $2.851 \mathrm{c}$ & $1.829 \mathrm{~b}$ & $2.321 \mathrm{~b}$ & $2.699 \mathrm{~b}$ \\
\hline 25 & & $2.083 \mathrm{c}$ & $2.329 \mathrm{~d}$ & $2.534 d$ & $1.687 \mathrm{c}$ & $1.982 \mathrm{c}$ & $2.258 \mathrm{c}$ \\
\hline $\operatorname{LSD}_{0.05}$ & & 0.062 & 0.013 & 0.018 & 0.026 & 0.022 & 0.043 \\
\hline \multicolumn{8}{|c|}{ B- Humic acid: } \\
\hline \multirow{5}{*}{\multicolumn{2}{|c|}{$\begin{array}{l}\text { Without } \\
\text { Seed soaking } \\
\text { Soil application } \\
\text { Foliar spraying } \\
\text { LSD }_{0.05} \\
\end{array}$}} & $2.112 \mathrm{~d}$ & $2.301 \mathrm{~d}$ & $2.485 \mathrm{~d}$ & $1.638 \mathrm{~d}$ & $1.915 \mathrm{~d}$ & $2.179 \mathrm{~d}$ \\
\hline & & $2.255 \mathrm{c}$ & $2.490 \mathrm{c}$ & $2.677 \mathrm{c}$ & $1.766 \mathrm{c}$ & $2.073 \mathrm{c}$ & $2.480 \mathrm{c}$ \\
\hline & & $2.463 \mathrm{~b}$ & $2.711 b$ & $3.017 b$ & $1.879 \mathrm{~b}$ & $2.479 \mathrm{~b}$ & $2.775 \mathrm{~b}$ \\
\hline & & $2.541 \mathrm{a}$ & $2.864 \mathrm{a}$ & $3.157 \mathrm{a}$ & $2.036 \mathrm{a}$ & $2.592 \mathrm{a}$ & $3.121 \mathrm{a}$ \\
\hline & & & 0.02 & & & & \\
\hline \multicolumn{8}{|c|}{ C- Interaction: } \\
\hline \multirow[t]{4}{*}{100} & Without & 2.219 hi & $2.427 \mathrm{f}$ & $2.693 \mathrm{~g}$ & $1.782 \mathrm{e}$ & $1.999 \mathrm{f}$ & $2.430 \mathrm{~g}$ \\
\hline & Seed soaking & 2.481 ef & $2.811 \mathrm{c}$ & $2.977 \mathrm{~d}$ & $1.987 \mathrm{c}$ & $2.189 \mathrm{~d}$ & $2.696 \mathrm{e}$ \\
\hline & Soil application & $2.610 \mathrm{bc}$ & $2.871 \mathrm{~b}$ & $3.184 \mathrm{~b}$ & $2.034 \mathrm{c}$ & $2.700 \mathrm{~b}$ & $3.003 \mathrm{c}$ \\
\hline & Foliar spraying & $2.709 \mathrm{a}$ & $3.054 \mathrm{a}$ & $3.357 \mathrm{a}$ & $2.175 \mathrm{a}$ & $2.826 \mathrm{a}$ & $3.359 \mathrm{a}$ \\
\hline \multirow[t]{4}{*}{75} & Without & $2.139 \mathrm{j}$ & $2.351 \mathrm{~g}$ & $2.544 \mathrm{j}$ & $1.617 \mathrm{~h}$ & $1.947 \mathrm{fg}$ & $2.199 \mathrm{~h}$ \\
\hline & Seed soaking & $2.259 \mathrm{gh}$ & $2.406 \mathrm{f}$ & $2.602 \mathrm{i}$ & $1.711 \mathrm{f}$ & $2.159 \mathrm{~d}$ & $2.679 \mathrm{e}$ \\
\hline & Soil application & $2.585 \mathrm{~cd}$ & $2.869 \mathrm{~b}$ & $3.118 c$ & $1.893 \mathrm{~d}$ & $2.554 \mathrm{c}$ & $2.815 \mathrm{~d}$ \\
\hline & Foliar spraying & $2.664 a b$ & $3.089 \mathrm{a}$ & $3.330 \mathrm{a}$ & $2.016 \mathrm{c}$ & $2.649 \mathrm{~b}$ & $3.210 \mathrm{~b}$ \\
\hline \multirow[t]{4}{*}{50} & Without & $2.199 \mathrm{i}$ & $2.397 \mathrm{f}$ & 2.642 hi & 1.594 hi & $1.926 \mathrm{~g}$ & $2.187 \mathrm{~h}$ \\
\hline & Seed soaking & $2.305 \mathrm{~g}$ & $2.422 \mathrm{f}$ & $2.664 \mathrm{gh}$ & 1.727 f & $2.095 \mathrm{e}$ & $2.566 \mathrm{f}$ \\
\hline & Soil application & $2.443 \mathrm{f}$ & $2.670 \mathrm{~d}$ & $3.011 \mathrm{~d}$ & $1.904 \mathrm{~d}$ & $2.566 \mathrm{c}$ & $2.794 \mathrm{~d}$ \\
\hline & Foliar spraying & $2.537 \mathrm{de}$ & $2.778 \mathrm{c}$ & $3.086 \mathrm{c}$ & $2.089 \mathrm{~b}$ & $2.697 \mathrm{~b}$ & $3.250 \mathrm{~b}$ \\
\hline \multirow[t]{4}{*}{25} & Without & $1.888 \mid$ & $2.029 \mathrm{~h}$ & 2.061 I & $1.560 \mathrm{i}$ & $1.786 \mathrm{~h}$ & $1.903 \mathrm{i}$ \\
\hline & Seed soaking & $1.975 \mathrm{k}$ & $2.322 \mathrm{~g}$ & $2.465 \mathrm{k}$ & $1.638 \mathrm{gh}$ & $1.847 \mathrm{~h}$ & $1.980 \mathrm{i}$ \\
\hline & Soil application & 2.214 hi & $2.432 \mathrm{f}$ & $2.755 \mathrm{f}$ & $1.685 \mathrm{fg}$ & $2.096 \mathrm{e}$ & $2.486 \mathrm{~g}$ \\
\hline & Foliar spraying & 2.255 ghi & $2.535 \mathrm{e}$ & $2.855 \mathrm{e}$ & $1.864 \mathrm{~d}$ & $2.197 \mathrm{~d}$ & $2.664 \mathrm{e}$ \\
\hline LSD $_{0.05}$ & & 0.058 & 0.041 & & 0.051 & 0.062 & 0.079 \\
\hline
\end{tabular}

In each factor means followed by a common letter are not significantly different at the $5 \%$ level by LSD.

Increasing NPK rate from 25 to $100 \%$ of recommended rate resulted in a significant increase in LAI and dry weight/plant, CGR, plant height and number of fruiting branches/plant at all the mentioned sampling dates in the two seasons. The rate of $100 \%$ NPK recorded the highest values of the mentioned traits, while the rate of $25 \%$ NPK recorded the lowest ones in both seasons. However, application of 100 and $75 \%$ NPK, being insignificant, 
Seadh, S. E. et al.

exceeded application of $50 \%$ NPK in NAR at the first period in the two seasons (Table 6).

Table 4: Total dry weight (g/plant) as affected by NPK rates, humic acid treatments and their interaction in 2010 and 2011 seasons.

\begin{tabular}{|c|c|c|c|c|c|c|c|}
\hline \multirow{3}{*}{\multicolumn{2}{|c|}{ Treatments }} & \multirow{2}{*}{\multicolumn{3}{|c|}{$\begin{array}{c}2010 \text { season } \\
\text { Days from sowina }\end{array}$}} & \multicolumn{3}{|c|}{2011 season } \\
\hline & & & & & \multicolumn{3}{|c|}{ Days from sowing } \\
\hline & & 79 & 100 & 121 & 79 & 100 & 121 \\
\hline \multicolumn{8}{|c|}{ A- NPK rates: } \\
\hline 100 & & $49.90 \mathrm{a}$ & 89.90 a & $140.1 \mathrm{a}$ & $47.70 \mathrm{a}$ & $81.70 \mathrm{a}$ & $133.1 \mathrm{a}$ \\
\hline 75 & & $47.40 \mathrm{~b}$ & $84.30 \mathrm{~b}$ & $130.7 b$ & $44.40 \mathrm{~b}$ & $76.40 \mathrm{~b}$ & $123.0 \mathrm{~b}$ \\
\hline 50 & & $46.50 \mathrm{c}$ & $77.50 \mathrm{c}$ & $123.5 \mathrm{c}$ & $44.20 \mathrm{~b}$ & $74.50 \mathrm{c}$ & $121.0 \mathrm{~b}$ \\
\hline $\begin{array}{l}25 \\
\operatorname{LSD}_{0.05}\end{array}$ & & $\begin{array}{c}40.50 \mathrm{~d} \\
0.7\end{array}$ & $\begin{array}{c}71.00 \mathrm{~d} \\
3.4\end{array}$ & $\begin{array}{c}109.8 \mathrm{~d} \\
3.0\end{array}$ & $\begin{array}{c}38.50 \mathrm{c} \\
1.3\end{array}$ & $\begin{array}{c}66.50 \mathrm{~d} \\
1.3\end{array}$ & $\begin{array}{c}106.0 \mathrm{c} \\
\mathbf{2 . 1}\end{array}$ \\
\hline \multicolumn{8}{|c|}{ B- Humic acid: } \\
\hline Without & & $40.40 \mathrm{~d}$ & $64.90 \mathrm{~d}$ & $103.6 \mathrm{~d}$ & $37.40 \mathrm{~d}$ & $63.80 \mathrm{~d}$ & $103.3 \mathrm{c}$ \\
\hline Seed soc & & $44.50 \mathrm{c}$ & $78.30 \mathrm{c}$ & $124.6 \mathrm{c}$ & $41.30 \mathrm{c}$ & $70.80 \mathrm{c}$ & $115.0 \mathrm{~b}$ \\
\hline Soil appl & tion & $48.40 \mathrm{~b}$ & $86.60 \mathrm{~b}$ & $135.1 \mathrm{~b}$ & $46.20 \mathrm{~b}$ & $79.30 \mathrm{~b}$ & $131.2 \mathrm{a}$ \\
\hline $\begin{array}{l}\text { Foliar sp } \\
\text { LSD }_{0.05}\end{array}$ & ing & $\begin{array}{c}51.10 \mathrm{a} \\
1.5\end{array}$ & $\begin{array}{c}93.00 \mathrm{a} \\
2.2\end{array}$ & $\begin{array}{c}140.8 \mathrm{a} \\
2.2\end{array}$ & $\begin{array}{c}49.90 \mathrm{a} \\
\mathbf{1 . 6}\end{array}$ & $\begin{array}{c}85.20 \mathrm{a} \\
1.7\end{array}$ & $\begin{array}{c}133.4 \mathrm{a} \\
2.2\end{array}$ \\
\hline \multicolumn{8}{|c|}{ C- Interaction: } \\
\hline \multirow[t]{4}{*}{100} & Without & 44.30 ef & $74.50 \mathrm{f}$ & $121.6 \mathrm{fg}$ & 41.0 & $70.60 \mathrm{hi}$ & $114.5 \mathrm{e}$ \\
\hline & Seed soaking & $48.10 \mathrm{~cd}$ & $86.50 \mathrm{c}$ & $135.1 \mathrm{~d}$ & 44.1 & $75.40 \mathrm{fg}$ & $126.2 \mathrm{c}$ \\
\hline & Soil application & $52.20 \mathrm{ab}$ & $93.30 \mathrm{~b}$ & $149.1 \mathrm{~b}$ & 50.4 & $88.40 \mathrm{~b}$ & $145.4 \mathrm{a}$ \\
\hline & Foliar spraying & $54.90 \mathrm{a}$ & $105.3 \mathrm{a}$ & $154.5 \mathrm{a}$ & 55.2 & $92.40 \mathrm{a}$ & $146.5 \mathrm{a}$ \\
\hline \multirow[t]{4}{*}{75} & Without & $42.10 \mathrm{fg}$ & $63.00 \mathrm{~h}$ & $104.9 \mathrm{i}$ & 39.1 & $69.00 \mathrm{i}$ & $108.0 \mathrm{f}$ \\
\hline & Seed soaking & 44.60 ef & 80.10 de & $125.0 \mathrm{f}$ & 42.5 & $73.30 \mathrm{gh}$ & $116.0 \mathrm{e}$ \\
\hline & Soil application & $49.90 \mathrm{bc}$ & $92.40 \mathrm{~b}$ & $139.5 c$ & 46.4 & $79.70 \mathrm{e}$ & $130.6 \mathrm{c}$ \\
\hline & Foliar spraying & $53.10 \mathrm{a}$ & $101.7 \mathrm{a}$ & $153.5 \mathrm{a}$ & 49.5 & $83.50 \mathrm{~cd}$ & $136.2 b$ \\
\hline \multirow[t]{4}{*}{50} & Without & 43.90 ef & $69.30 \mathrm{~g}$ & $110.6 \mathrm{~h}$ & 39.0 & $65.00 \mathrm{j}$ & $108.6 \mathrm{f}$ \\
\hline & Seed soaking & $46.00 \mathrm{de}$ & 76.50 ef & $120.8 \mathrm{fg}$ & 41.1 & $70.30 \mathrm{hi}$ & $116.1 \mathrm{e}$ \\
\hline & Soil application & $46.40 \mathrm{de}$ & $80.20 \mathrm{de}$ & $129.6 \mathrm{e}$ & 46.8 & 78.40 ef & $130.4 \mathrm{c}$ \\
\hline & Foliar spraying & $49.80 \mathrm{bc}$ & $84.10 \mathrm{~cd}$ & $133.1 \mathrm{de}$ & 50.1 & $84.20 \mathrm{c}$ & $129.5 \mathrm{c}$ \\
\hline \multirow[t]{3}{*}{25} & Without & $31.30 \mathrm{~h}$ & $52.60 \mathrm{i}$ & $77.40 \mathrm{j}$ & 30.6 & $50.60 \mathrm{k}$ & $82.20 \mathrm{~h}$ \\
\hline & Seed soaking & $39.20 \mathrm{~g}$ & $70.00 \mathrm{~g}$ & $117.5 \mathrm{~g}$ & 37.3 & $64.30 \mathrm{j}$ & $101.8 \mathrm{~g}$ \\
\hline & Soil application & 45.00 ef & $80.40 \mathrm{de}$ & $122.1 \mathrm{f}$ & 41.3 & $70.60 \mathrm{hi}$ & $118.7 \mathrm{de}$ \\
\hline $\operatorname{LSD}_{0.05}$ & Foliar spraying & $\begin{array}{l}46.60 \mathrm{de} \\
2.96\end{array}$ & $\begin{array}{c}81.00 \mathrm{~d} \\
4.47\end{array}$ & $\begin{array}{c}122.2 f \\
4.36\end{array}$ & $\begin{array}{l}44.9 \\
\text { NS }\end{array}$ & $\begin{array}{c}80.60 \mathrm{de} \\
3.49\end{array}$ & $\begin{array}{c}121.5 \mathrm{~d} \\
4.44\end{array}$ \\
\hline
\end{tabular}

In each factor means followed by a common letter are not significantly different at the $5 \%$ level by LSD

The rates of 100,75 and $25 \%$ NPK were statistically at par in NAR in the two seasons. The increase in growth with increasing nitrogen rate could be attributed mainly to $\mathrm{N}$ role in the stimulation of various physiological processes including cell division and cell elongation resulting in more photosynthetic area (leaf area), which resulted in more photosynthetic production and consequently increased dry matter accumulation, CGR , plant height and number of fruiting branches. Also, phosphorus application might be increasing phosphorus availability and subsequently increased its uptake and its content in cotton plants leading to more ATP formation, that is the main stare for energy in plant and might encourage cotton growth, metabolism, photosynthesis and nucleic acid resulted in high efficiency of dry matter production. Application of $\mathrm{K}$ enhanced the enzymatic activities, probably caused higher mobilization of nutrients in soil and plant and 
translocation of photosynthetic in plant system, which ultimately resulted in higher dry matter accumulation. The promoting effects of nitrogen on cotton growth were reported by Jia et al. (1985), Chhabra and Bishnoi (1993), El Beily et al. (2001), El- Shazly and Darwish (2001), Shriram and Prasad (2001), Zhao and Oosterhuis (2001) and El- Shazly (2011), who found that LAl increased with increasing N levels. Hearn (1981), Cadena and Cothren (1995) and Hutmacher et al. (1995) indicated that adding N fertilizer had a positive effect on photosynthesis rates. Zhao and Oosterhuis (2001) found that low $\mathrm{N}$ decreased leaf net photosynthetic rate during fruiting than the high $\mathrm{N}$ treatment. (Uchida, 2000) stated that $\mathrm{P}$ is required in large quantities in young cells, such as shoots and root tips, where metabolism is high and cell division is rapid. Also, Because $\mathrm{K}$ is needed in photosynthesis and the synthesis of protein, plants lacking $\mathrm{K}$ will have slow and stunted growth (Uchida, 2000). Srinivasan (2006) found that plant height increased by increasing NPK rate.

Table 5: Crop growth rate $\left(\mathrm{g} / \mathrm{m}^{2} /\right.$ week) as affected by NPK rates, humic acid treatments and their interaction in 2010 and 2011 seasons.

\begin{tabular}{|c|c|c|c|c|c|}
\hline \multirow{3}{*}{\multicolumn{2}{|c|}{ Treatments }} & \multirow{2}{*}{\multicolumn{2}{|c|}{$\begin{array}{c}2010 \text { season } \\
\text { Days from sowing }\end{array}$}} & \multicolumn{2}{|c|}{2011 season } \\
\hline & & & & Days frc & sowing \\
\hline & & $79-100$ & $100-121$ & $79-100$ & $100-121$ \\
\hline \multicolumn{6}{|c|}{ A- NPK rates: } \\
\hline 100 & & $152.6 \mathrm{a}$ & $191.0 \mathrm{a}$ & $129.6 \mathrm{a}$ & $195.9 \mathrm{a}$ \\
\hline 75 & & $140.5 b$ & $176.8 \mathrm{a}$ & $121.9 \mathrm{~b}$ & $177.5 \mathrm{~b}$ \\
\hline 50 & & $118.2 \mathrm{c}$ & $175.2 \mathrm{a}$ & $115.2 \mathrm{c}$ & $177.1 \mathrm{~b}$ \\
\hline & & $116.2 \mathrm{c}$ & $147.8 \mathrm{~b}$ & $106.7 \mathrm{~d}$ & $150.6 \mathrm{c}$ \\
\hline LSD & & 6.4 & 20.3 & 5.3 & 15.2 \\
\hline \multicolumn{6}{|c|}{ B- Humic acid: } \\
\hline \multirow{5}{*}{\multicolumn{2}{|c|}{$\begin{array}{l}\text { Without } \\
\text { Seed soaking } \\
\text { Soil application } \\
\text { Foliar spraying } \\
\text { LSD }_{0.05} \\
\end{array}$}} & $93.20 \mathrm{~d}$ & $147.6 \mathrm{~b}$ & $100.5 \mathrm{~d}$ & $150.5 \mathrm{c}$ \\
\hline & & $128.9 \mathrm{c}$ & $176.4 \mathrm{a}$ & $112.6 \mathrm{c}$ & $168.4 \mathrm{~b}$ \\
\hline & & $145.6 \mathrm{~b}$ & $184.8 \mathrm{a}$ & $125.9 b$ & $197.8 \mathrm{a}$ \\
\hline & & $159.8 \mathrm{a}$ & $182.0 \mathrm{a}$ & $134.4 \mathrm{a}$ & $183.8 \mathrm{a}$ \\
\hline & & 9.9 & 8.4 & 7.9 & 14.1 \\
\hline \multicolumn{6}{|c|}{ C- Interaction: } \\
\hline \multirow[t]{4}{*}{100} & Without & 115.3 ef & 179.2 cde & $112.6 \mathrm{fgh}$ & 167.2 \\
\hline & Seed soaking & $146.5 \mathrm{bc}$ & $184.9 \mathrm{~b}-\mathrm{e}$ & 119.1 def & 193.5 \\
\hline & Soil application & $156.7 \mathrm{~b}$ & $212.3 \mathrm{a}$ & $144.7 \mathrm{a}$ & 217.0 \\
\hline & Foliar spraying & $192.1 \mathrm{a}$ & $187.4 \mathrm{bcd}$ & $141.9 \mathrm{ab}$ & 206.0 \\
\hline \multirow[t]{4}{*}{75} & Without & $79.80 \mathrm{~g}$ & $159.3 \mathrm{f}$ & 114.1 e-h & 148.6 \\
\hline & Seed soaking & $135.2 \mathrm{~cd}$ & 171.0 def & $117.1 \mathrm{~d}-\mathrm{g}$ & 162.9 \\
\hline & Soil application & $161.6 \mathrm{~b}$ & 179.5 cde & $126.9 b-f$ & 194.0 \\
\hline & Foliar spraying & $185.3 \mathrm{a}$ & $197.2 \mathrm{ab}$ & 129.6 a-e & 200.8 \\
\hline \multirow[t]{4}{*}{50} & Without & $96.60 \mathrm{fg}$ & $157.4 \mathrm{f}$ & $99.10 \mathrm{~h}$ & 166.1 \\
\hline & Seed soaking & $116.5 \mathrm{de}$ & 168.6 ef & $111.4 \mathrm{fgh}$ & 174.5 \\
\hline & Soil application & 128.9 cde & $188.3 \mathrm{bc}$ & $120.3 c-f$ & 198.2 \\
\hline & Foliar spraying & 130.7 cde & $186.6 \mathrm{bcd}$ & $130.1 \mathrm{a}-\mathrm{d}$ & 172.4 \\
\hline \multirow[t]{5}{*}{25} & Without & $81.10 \mathrm{~g}$ & $94.40 \mathrm{~g}$ & $76.20 \mathrm{i}$ & 120.3 \\
\hline & Seed soaking & $117.3 \mathrm{de}$ & $181.1 \mathrm{~b}-\mathrm{e}$ & $102.9 \mathrm{gh}$ & 142.7 \\
\hline & Soil application & $135.1 \mathrm{~cd}$ & $159.0 \mathrm{f}$ & $111.8 \mathrm{fgh}$ & 183.2 \\
\hline & Foliar spraying & 131.2 cde & $156.7 \mathrm{f}$ & $135.9 \mathrm{abc}$ & 156.1 \\
\hline & & 19.78 & 16.82 & 15.77 & NS \\
\hline
\end{tabular}

NS indicates not significant. In each factor means followed by a common letter are not significantly different at the $5 \%$ level by LSD. 
Seadh, S. E. et al.

\section{2- Effect of humic acid}

Application of humic acid solution resulted in a significant increase in all mention growth measurements (except NAR) compared with control treatment in both seasons (Tables 3, 4, 5, 6,7).

Table 6: Net assimilation rate $\left(\mathrm{g} / \mathrm{m}^{2} /\right.$ week) as affected by NPK rates, humic acid treatments and their interaction in 2010 and 2011 seasons.

\begin{tabular}{|c|c|c|c|c|c|}
\hline & \multirow{3}{*}{ Treatments } & \multicolumn{2}{|c|}{2010 season } & \multicolumn{2}{|c|}{2011 season } \\
\hline & & \multicolumn{2}{|c|}{ Days from sowing } & \multicolumn{2}{|c|}{ Days from sowing } \\
\hline & & $79-100$ & $100-121$ & $79-100$ & $100-121$ \\
\hline \multicolumn{6}{|c|}{ A- NPK rates: } \\
\hline 100 & & $57.20 \mathrm{a}$ & 65.6 & $58.70 \mathrm{a}$ & 74.55 \\
\hline 75 & & $54.20 \mathrm{a}$ & 63.7 & $59.30 \mathrm{a}$ & 70.11 \\
\hline & & $47.70 \mathrm{~b}$ & 64.7 & $55.70 \mathrm{~b}$ & 72.04 \\
\hline & & $52.20 \mathrm{ab}$ & 60.4 & $57.70 \mathrm{ab}$ & 71.11 \\
\hline $\operatorname{LSD}_{0.05}$ & & 5.3 & NS & 2.2 & NS \\
\hline \multicolumn{6}{|c|}{ B- Humic acid: } \\
\hline \multirow{5}{*}{\multicolumn{2}{|c|}{$\begin{array}{l}\text { Without } \\
\text { Seed soaking } \\
\text { Soil application } \\
\text { Foliar spraying } \\
\text { LSD }_{0.05}\end{array}$}} & $42.20 \mathrm{c}$ & $60.90 \mathrm{c}$ & 56.4 & $73.30 \mathrm{a}$ \\
\hline & & $54.30 \mathrm{~b}$ & $68.60 \mathrm{a}$ & 58.7 & $74.00 \mathrm{a}$ \\
\hline & & $56.30 \mathrm{ab}$ & $64.50 \mathrm{~b}$ & 57.8 & $75.40 \mathrm{a}$ \\
\hline & & 58.70 a & $60.40 \mathrm{c}$ & 58.4 & $64.30 \mathrm{~b}$ \\
\hline & & 4.1 & 3.3 & & 4.1 \\
\hline \multicolumn{6}{|c|}{ C- Interaction: } \\
\hline \multirow[t]{4}{*}{100} & Without & 49.60 ef & $70.00 a b$ & $59.60 \mathrm{bcd}$ & 75.74 \\
\hline & Seed soaking & 55.40 cde & $63.90 \mathrm{~b}-\mathrm{e}$ & $57.00 \mathrm{bcd}$ & 79.52 \\
\hline & Soil application & $57.20 \mathrm{~b}-\mathrm{e}$ & $70.10 \mathrm{ab}$ & $61.10 \mathrm{abc}$ & 76.17 \\
\hline & Foliar spraying & $66.70 \mathrm{a}$ & $58.50 \mathrm{e}$ & $56.90 \mathrm{bcd}$ & 66.77 \\
\hline \multirow[t]{4}{*}{75} & Without & $35.50 \mathrm{~h}$ & $65.10 \mathrm{bcd}$ & $64.00 \mathrm{ab}$ & 71.76 \\
\hline & Seed soaking & $57.90 \mathrm{bcd}$ & $68.30 \mathrm{bc}$ & 60.50 a-d & 67.62 \\
\hline & Soil application & $59.30 \mathrm{abc}$ & $60.00 \mathrm{de}$ & $57.00 \mathrm{bcd}$ & 72.32 \\
\hline & Foliar spraying & $64.30 \mathrm{ab}$ & $61.50 \mathrm{de}$ & $55.50 \mathrm{~cd}$ & 73.59 \\
\hline \multirow[t]{4}{*}{50} & Without & $42.00 \mathrm{fgh}$ & 62.50 cde & $56.30 \mathrm{~cd}$ & 80.87 \\
\hline & Seed soaking & 49.30 efg & $66.40 \mathrm{bcd}$ & $58.30 \mathrm{bcd}$ & 75.14 \\
\hline & Soil application & $50.40 \mathrm{de}$ & $66.30 \mathrm{bcd}$ & $53.80 \mathrm{~d}$ & 73.99 \\
\hline & Foliar spraying & 49.20 efg & $63.70 \mathrm{~b}-\mathrm{e}$ & $54.40 \mathrm{~cd}$ & 58.15 \\
\hline \multirow[b]{4}{*}{ on } & Without & $41.40 \mathrm{gh}$ & $46.20 \mathrm{f}$ & $45.50 \mathrm{e}$ & 65.22 \\
\hline & Seed soaking & 54.60 cde & $75.80 \mathrm{a}$ & $59.10 \mathrm{bcd}$ & 74.63 \\
\hline & Soil application & $58.10 \mathrm{bcd}$ & $61.70 \mathrm{de}$ & $59.10 \mathrm{bcd}$ & 80.14 \\
\hline & Foliar spraying & $\begin{array}{l}54.80 \text { cde } \\
812\end{array}$ & $58.20 \mathrm{e}$ & $66.90 \mathrm{a}$ & $\begin{array}{c}64.43 \\
\text { NS }\end{array}$ \\
\hline $\operatorname{LSD}_{0.05}$ & & 8.12 & 6.56 & 1.2 & NS \\
\hline
\end{tabular}

NS indicates not significant. In each factor means followed by a common letter are not significantly different at the $5 \%$ level by LSD.

Data show that foliar application of humic acid at three time (at 40,55 and 70 DFS) was superior to soil application twice and soaking seed for $18 \mathrm{~h}$ in LAI, total dry weight/plant, CGR, plant height and number of fruiting branches/plant in the two seasons. Soil application of humic acid surpassed soaking seed in all the mention growth traits in both seasons. Humic acid is one of the major components of humus. Humates are natural organic substances, high in humic acid and containing most of known trace minerals (Table 2) necessary to the development of plant life(Senn, 1991). Also, Trace minerals have a vital role in improve cotton growth, where it works as coenzyme and as activators and enhanced amino acid formation, subsequently 
increased photosynthesis leading to high dry matter production. In this concern, Emara and Hamoda (2012) found that foliar spraying of humex 5 $\mathrm{cm}^{3} / \mathrm{L}$ three times produced the tallest plants and the greatest number of sympodia/plant.

The relative ranking of humic acid treatments for NAR was inconsistent at the two period in the first season and the second period in the second season (Table 6). Plants of seed soaking in solution of humic acid sol were among those having high NAR at the second period in both seasons. However, foliar application of humic acid produced the lowest NAR in this period in the tow seasons. This may be due to the increase in mutual shading and/or the dilution effect caused by the large leaf area formed by application of humic acid, which in turn decreased NAR.

Table 7: Plant height and number of fruiting branches as affected by NPK rates, humic acid treatments and their interaction in 2010 and 2011 seasons.

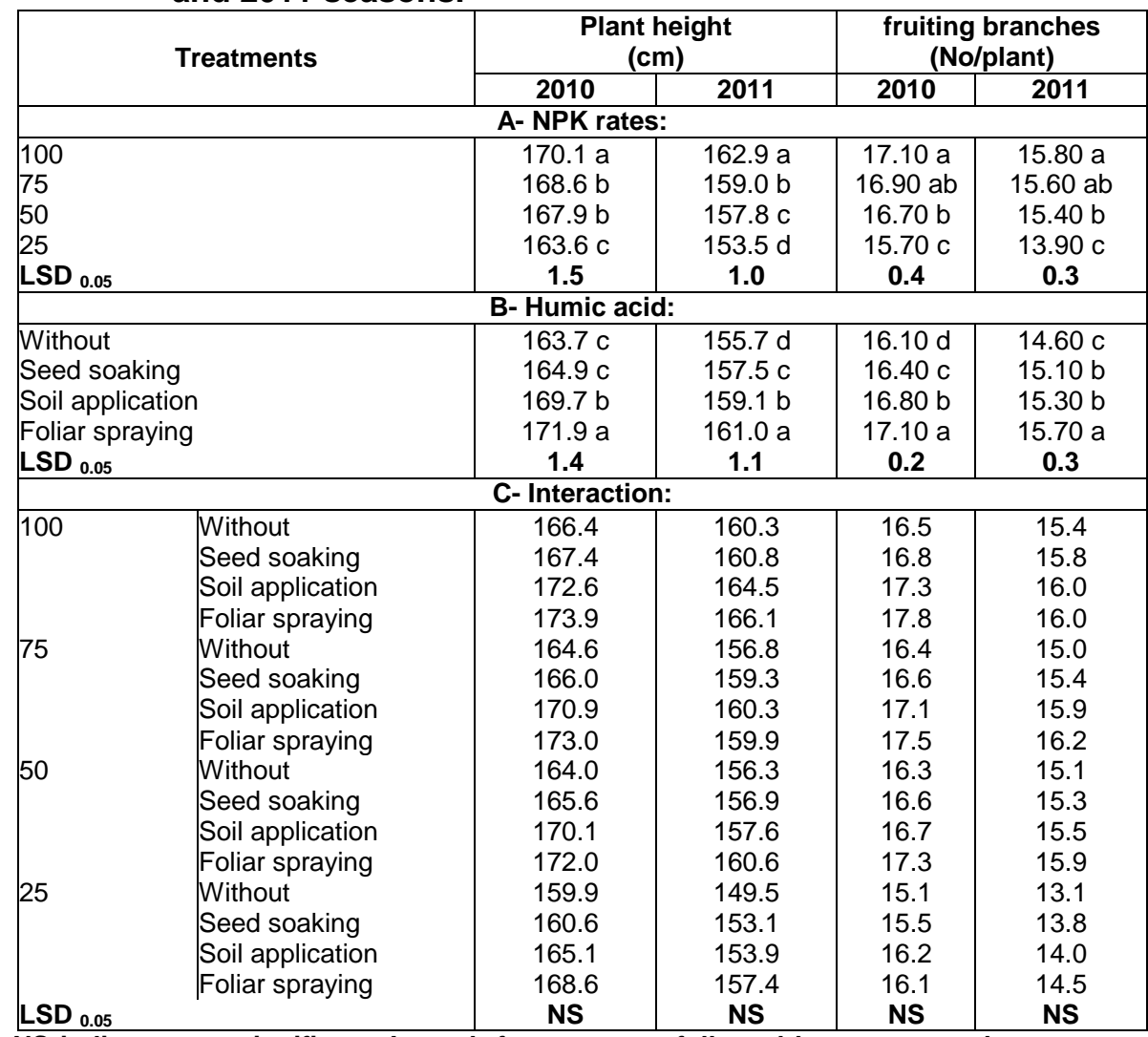

NS indicates not significant. In each factor means followed by a common letter are not significantly different at the $5 \%$ level by LSD. 


\section{3- Effect of the interaction:}

LAl, dry weight/plant (except at 79 DFS in the second season) as well as CGR and NAR (except at the second period in the second season) were significantly influenced by the interaction between NPK rate and application methods of humic acid in the two seasons (Tables 3, 4, 5 and 6). Data in Tables 3, 4, 5 show that plants received 100\% NPK along with foliar spraying with humic acid produced higher LAI, dry weight/plant and CGR than those received $25 \%$ NPK without humic acid application at the most sampling date in both seasons. There were no significant differences among the combination of 100 or $75 \%$ NPK and foliar application of humic acid in $\mathrm{LAl}$, dry weight/plant and CGR in the first season.

The relative ranking of the interaction between NPK rate and application methods of humic acid for NAR was inconsistent at the two period in both seasons (Table 6). The highest NAR was obtained from the combination of $100 \%$ NPK and foliar application of humic acid at the first period in the first season, the combination of $25 \%$ NPK and seed soaking in humic acid solution at the second period in the first season and the combination of $25 \%$ NPK and foliar application of humic acid at the first period in the second season.

None of the interaction had a significant effect on plant height and number of fruiting branches/plant in the two seasons (Table 7).

\section{Conclusion}

It can be concluded that application rate of $75 \%$ NPK in combined with humic acid (in the form of actosol) as foliar application at three time (40, 55 and 70 DFS) could be recommended for optimum growth of Egyptian cotton cv. Giza 86 at Gemmeiza condition. This result indicated that foliar application of humic acid can be saved $25 \%$ NPK fertilizer without reduce the growth of cotton plants.

\section{REFERENCES}

Basbag, S. (2008). Effects of humic acid application on yield and quality of cotton. (Gossybium hrisutum L. ). Asian J .of chemistry. 20(3) : 19611966.

Blevins,D.G. and K.M. Lukaszewski (1998) Boron in plant structure and function. Annu .Rev.plant physiol. Plant Mol.Biol.1998, 49:481-500.

Cadena,J. and J .T. Cothren, (1995). Yield response of cotton to nitrogen, irrigation and PGR - IV regimes, Proce. Beltwide Cotton Conf. , San Antonio, TX, USA, Jan., 4-7, 2:1142-1150 .

Chellaiah , N. ;U. Solaiappan and S. Senthivel. (2001). Studies on foliar nutrition on productivity of summer irrigated cotton. Madras Agric. J ., $88(1 / 3): 180-181$

Chhabra , K. L. and K. C. Bishnoi (1993). Response of American Cotton Varieties to plant spacings and nitrogen levels on growth characters. $J$ . Cotton Research and Development 7(1): 101-109 (C.F. Field Crop Abst. , 49(1):474,1996)

Dobermann, A. ; Cassman, K.G. ; Walters , D. T. and C, Witt (2005) Balancing Short - term and long-term goals in nutrient management . Better Crops with Plant Food. 89: 4, 16-19. 
Ebelhar, M. W and J. O. Ware (2003). Nitrogen management for mid - south cotton production over view . Beltwide Cotton Conference, Nashville, TN - January 6-10:159-164 .

El-Beily, M. A. A.; W. M. O. El - Shazly ; S. A. Ali and K. A. Ziadah (2001). Response of cotton cultivar Giza 85 to nitrogen rates and hill spacing under levels of growth regular (Pix) . Minufiya J. Agric . Res. ,26(1):5184.

El-Fouly, M. M. and A.F. Abd El- Hamid (1992). Principles of Plant Nutrition, fertilization and problems of micronutrients in Egypt. National Research Center, El- Dokki, Cairo . Protect of micronutrients and Problems of plant nutrition in Egypt, 3rd Ed. Pp;1-156 (In Arabic )

El-Gala, A. M. (1978). Methods of improving the fertility of sandy soils influence of organic substances . Egypt. J . Soil Sci. (Special Issuess ) : 57-60

El-Ganaini, S. S. ; M. A. Saif El- Yazal and S. E. A. Mohemed (2005). Botanical Studies on cotton (Gossypium barbadense, L. ) plants grown under newly reclaimed soils as affected by nitrogen and phosphorus fertilization. Annals of Agricultural Science, Moshtohor. 43: 4, 1599-1617

El-Sayed, E. A. and M. El- Menshawi (2005). Effect of mineral nitrogen and some bio-organic fertilizers on growth, yield andsome constituents of Giza 88 cotton cultivar . J. Agric. Res. Tanta Univ., 31(4-A):616-629.

El-Shazly , W. M. O. and M. F El-Masri (2002). A comparative study on the effect of some organic manures and mineral nitrogen fertilizersa on the leaf chemical composition, growth, earliness and yield of cotton (Giza 89 cultivar ). Minufiya, J. Agric. Res. , 27(6) :1325-1348.

El-Shazly, M. W. M. (2011).Bio-Chemical studies on cotton plant .M. Sc. Thesis, Fac. Agric.,Tanta Univ.

El-Shazly, W. M. O. and A. A. Darwish (2001)Response of cotton (Giza 89 cultivar ) to nitrogen levels and biofertilization with microbein . Minufiya J. Agric Res. , 26 (3) :635-658.

Emara ,M. A. and S. F. Hamoda (2012). Effect of humex on growth, yield and quality of cotton under calcareous soil conditions. Alexandria International Cotton Conference, Faculty of Alexandria, Saba Basha , Alex. ,Univ. Alex. $17^{\text {th }}-18^{\text {th }}, 2012$.

Fataftah ,A. K.;D. S. Watia ; B. G. Ains and S. I. Kotob (2001). Comparative evaluation of known liquid humic analysis method. Arctech Inc. ,Chantilly, V. A. 20165 ,U.S. A.

Fernandez , C. J.; K .J . Mcinnes and J. T. Cothren (1996) Water status and leaf area production in water and nitrogen stressed cotton. Crop Sci., $36(5): 1224-1233$.

Hearn, A.B . (1981). Cotton nutrition . Field Crop Abst. , 34 (1): 11-34.

Hull, H.M.; Morton, H.L. , and Wharrie , J.R.( 1975) . Environmental influence on cuticle development and resultant foliar penetration. Botanical Review, 41, $421-451$.

Hutmacher, R .B .; C . J . phene; K. R . Davis; S. S . Vail; T, P. Flaum; M. S. Peters; C .A. Hawk; D .A. Clark; A. D. Bravo; T. A. Kerby ; M . Keeley and D .A. Ballard ( 1995 ). Nitrogen uptake of Acalo and Pima cotton under high yield, drip irrigation conditions, Crop responses, effects of water deficits. Proc. Beltwide cotton conference, Sam Antonio, TX, USA, 4-7 Jan, 1995, Vol. 2:1295 - 1300 National Cotton Council, America.

Jackson, M. L. (1973). Soil Chemical Analysis. Prentice Hall of Indian Private Limited, New Delhi . 
Jio, R. Q . ; D. Z. Ye ; Y . M. Shi; G. Y. Cheng; C. G. Shi and X. Z. Shao (1985). Effects of nitrogen fertilization in the flowering and fruiting period on the growth and yield of cotton mulched plastic films. ZheJiang Agric. Sci. No. 4, 183 - (C.F. Field Crop Abst. 41(1): 661, 1988).

Johnson, R. E. (1967). Comparison of methods for estimating cotton leaf area. Agron. J. 59 (5): $493-494$.

Kalaichelvi, K. and Chinnusamy, C (2005). Influence of STCR based fertilizer nutrients and potassium humate on the productivity and profitability of Cotton - black gram cropping system. Research on Crops 6: 3, $477-$ 482.

McCauley, A; C. Jones and J. Jacobsen (2009) Plant nutrient functions and deficiency and Toxicity symptoms. Nutrient Management. Montana State Univ. Extension 4449 - 9 May 2009.

Radford's, P.J. (1967). Growth analysis formulae, their use and abuse. Crop Sci., 7: 171-175.

Reddy, P'.R .R .; Gopinath , M . and L . J . Rao ( 2007) Response of Bt cotton to nutrition and plant geometry . Journal of Research ANGRAU. 35: $4,53-54$.

Reddy, P .R .R . and Gopinath , M . (2008) . Influence of fertilizers and plant geometry on performance of Bt cotton hybrid .J . of cotton Research and Development . 22:1, 78-80 .

Senn, T.L. (1991). Humates in Ágriculture. Acres USA, Jan.

Shriram K. V. and M. Prasad (2001). Effect of nitrogen, bio fertilizer and growth regulator on growth, yield and quality of cotton. Fertiliser News, 46(5): $57-58$.

Snedecor, G. W. and W. G. Cochran (1981). Statistical Methods . lowa State Univ. Press 7th ed. lowa, USA.

Srinivasan, G . (2006) Agronomic evaluation of Bt cotton hybrids in summer irrigated tract of southern Tanil Nadu. J. of cotton Research and Development. 20: 2, $224-225$.

Srinivasan, G. and K. Venkatesan (2003). Response of summer irrigated cotton (Gossypium hirsutum L.) to reduce level of nutrients and time of application. Madras Agric. J., 90 (7-9): 528-529.

Taha, A.A. ; S.A. Modaish and M.O. Mahgoub (2006). Effect of some humic acids on wheat plant grown in different soils. J. Agric. Sci. Mansoura Univ., 31: (6): 4031-4039.

Uchida, R. ( 2000) . Essential nutrients for plant growth : nutrient functions and deficiency symptoms. Plant Nutrient Management in Hawaiis soils. Approaches for tropical and Subtropical Agriculture. Chapter 3: 31-55.

Zhao , D. and D. Oosterhuis (2001) . Cotton plant physiological and yield responses to nitrogen status. Proc. Beltwide Cotton Conference, Vol. 1: 511 - 514 National Cotton Council, Memphis I M. 


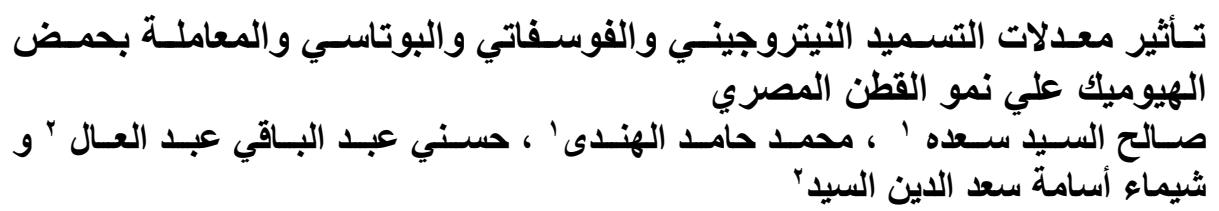

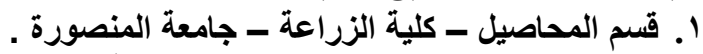

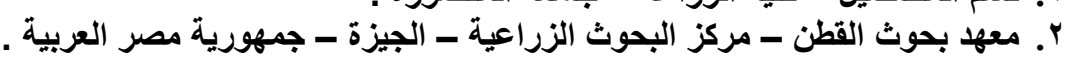

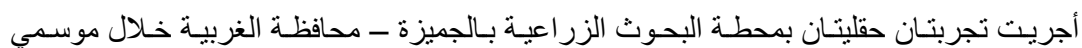

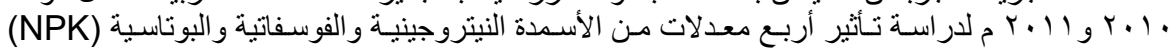

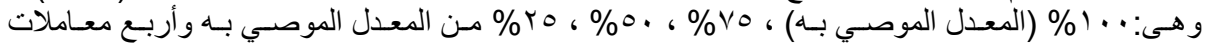

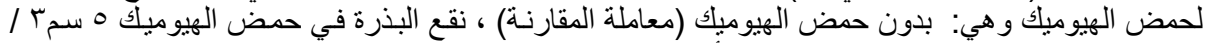

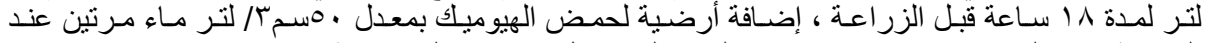

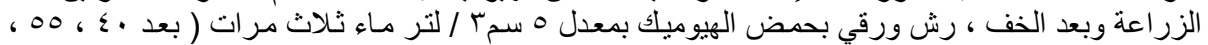

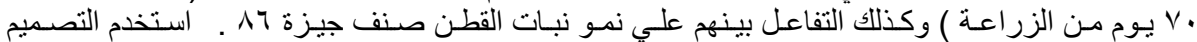

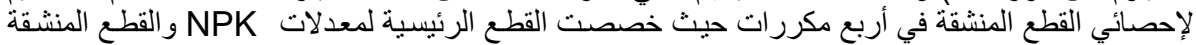

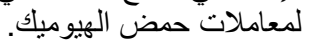

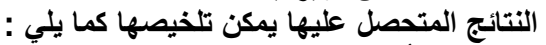

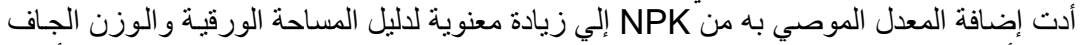

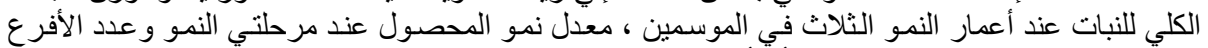

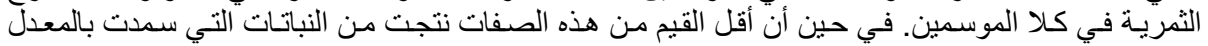

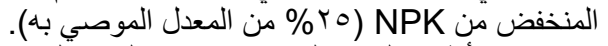

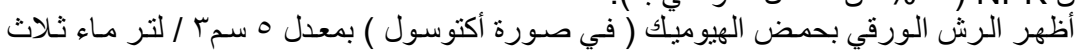

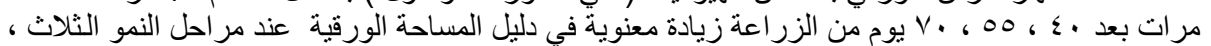

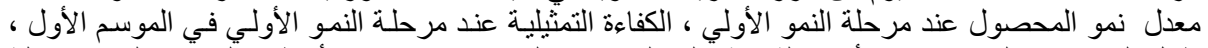

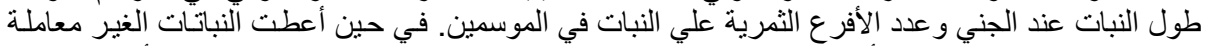

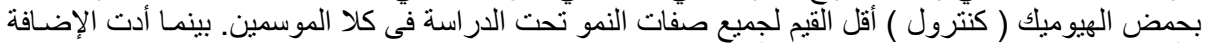

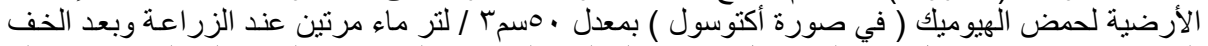

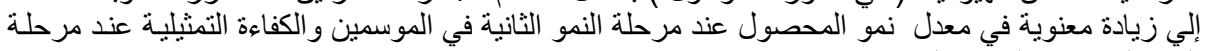
النمو الثانية في الموسم الثاني.

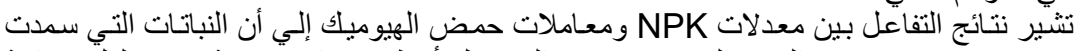

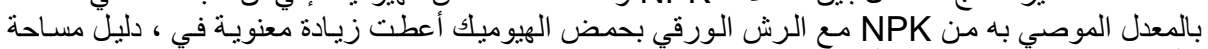

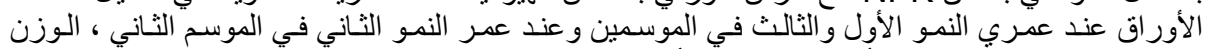

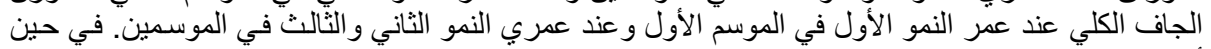

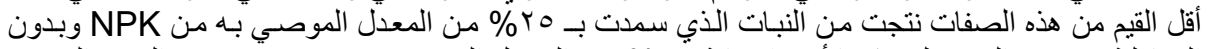

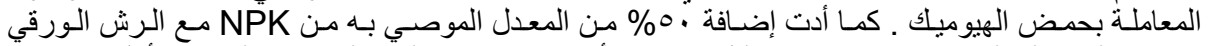

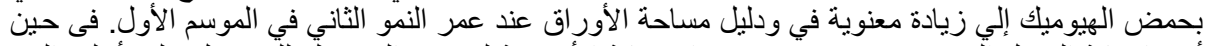

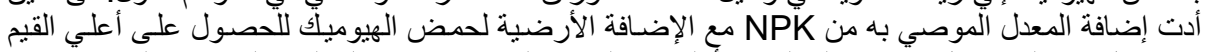

من معدل نمو المحصول عند مرحلة النمو الأولي في الإضي الموسم الثاني وعند مرحلة النمو الثانية في الموسمين.

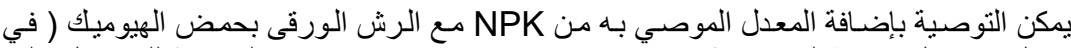

التوصية

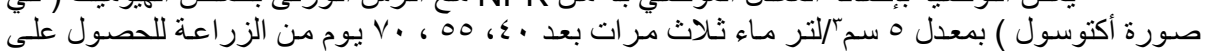

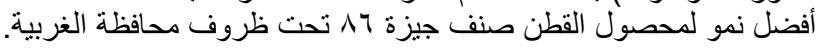

كلية الزراعة - جامعة المنصورة كلية الزراعة - جامعة كفر الثيخ

$$
\text { قام بتحكيم البحث }
$$

أ. أد / احمد نادر السيد عطيه

أ.د / صبحى غريب رزق سرور العبد عطيه 